\title{
Predictors of Acute Neurological Worsening after Endovascular Thrombectomy
}

\author{
Jazba Soomro Liang Zhu Sean I. Savitz Amrou Sarraj \\ Department of Neurology, McGovern Medical School at the University of Texas Health \\ Science Center at Houston, Houston, TX, USA
}

\section{Keywords}

Acute stroke $\cdot$ Thrombectomy $\cdot$ Endovascular therapy $\cdot$ Hyperglycemia $\cdot$ Acute worsening

\begin{abstract}
Background: Successful reperfusion after endovascular thrombectomy (EVT) correlates with good outcome. However, radiographic reperfusion does not always translate into good clinical outcomes even if the reperfusion occurs early after the stroke onset. Reasons for neurological worsening (NW) are thought to be many, such as progression of the stroke, hemorrhagic conversion post tissue plasminogen activator and/or EVT, and procedural complications such as vessel dissection or perforation, distal emboli, and re-occlusion. Data on patients worsening in the acute phase after EVT are limited. Objective: We studied the factors associated with acute NW and also identified the predictors of NW after EVT and its association with poor outcome at discharge. Methods: A retrospective cohort from a single comprehensive stroke center includes patients with acute ischemic stroke and large vessel occlusion in anterior and posterior circulation who presented between December 2014 and May 2017 and received EVT were reviewed. Primary outcome was defined as acute NW defined as change in NIHSS $\geq 4$ from baseline in the first $24 \mathrm{~h}$ after EVT. Secondary outcome were modified Rankin scale (mRS) $0-2$ at discharge and final infarct volume. Univariate and multivariate analyses were performed to evaluate clinical and radiographic variables independently correlating with NW after EVT. Receiver operating curve analysis was also performed to identify predictors. Results: 178 patients were included in the analysis, 26 (14.7\%) met the criteria for acute NW. For these 178 patients, the median age was 63 (IQR 53-74, range 26-89), baseline median NIHSS was 19 (IQR 14-24, range 5-37), ASPECTS was 8 (IQR 7-9, range 4-10), admission median systolic blood pressure (SBP) was 150 (IQR 131-170, range 94-287), and initial median blood glucose (BG) was 123 (IQR 106-157, range 69-433). The most common reasons for worsening were progression of the stroke $(42.3 \%)$ and reperfusion injury PH-2 $(26.9 \%)(p<0.0001)$.
\end{abstract}


Univariate logistic analysis showed that race, ASPECTS, collateral score, diabetes mellitus, admission SBP, and admission BG were associated with acute NW. In multivariate analysis, only admission BG (OR 1.00, Cl 1.00-1.01, $p=0.04)$ was found to have a significant association with acute NW. We ran a prediction analysis for variables and found the area under the curve to be 0.75 . Finally, there was strong association between NW and poor outcome at discharge (MRS 3-6, $p<0.01$ ) by Fisher's exact test. About $46.1 \%$ in the NW group died during hospitalization compared to $10 \%$ in the non-NW group $(p<0.0001)$. Conclusion: Our single-center retrospective cohort result is limited by small sample size. It showed that high admission BG is an independent predictor of NW after EVT and ultimately leads to poor outcome.

(c) 2019 S. Karger AG, Basel

\section{Introduction}

Endovascular thrombectomy (EVT) has been proven to be the more effective treatment in large vessel occlusion (LVO) than best medical management. It has been shown in prior studies that various clinical, radiographic, periprocedural, and intraprocedural factors and work flow metrics play a key role in achieving a timely successful reperfusion that translates into good clinical outcome [1-3]. Clinical improvement, i.e., improvement in baseline NIHSS, is often seen in the first $24 \mathrm{~h}$ after EVT [4, 5]. However, occasionally, despite achieving successful reperfusion (TICI $2 \mathrm{~b}$ or 3 ) in a timely fashion, some patients do not improve, and some even experience acute neurological worsening (NW) after EVT. NW in the acute phase of stroke or after thrombolysis has been defined in several ways in the literature; the most common definition used is $\geq 4$ points NIHSS from baseline in the first $24 \mathrm{~h}$. The prevalence of acute NW varies between 13 and 38\% after intravenous thrombolysis [6]. There are no good data on acute NW after EVT.

The possible reasons for deterioration after EVT have been postulated to be several, such as progression of the stroke, hemorrhagic conversion, re-occlusion, vessel perforation and infarction in the new territory, and distal embolization [7]. Hyperglycemia has been shown to be one of the modifiable factors that are commonly associated with unfavorable outcome in ischemic stroke patients who have been treated with IV tissue plasminogen activator (tPA). It has been linked to increase risk of symptomatic intracerebral hemorrhage, poor functional outcome, and less recanalization after IV tPA. No studies have yet reported the influence of hyperglycemia on the treatment effect of EVT. There is limited data on factors that are associated with acute (in the first $24 \mathrm{~h}$ ) NW after EVT, which in turn leads to poor outcome. The aim of this study was to identify predictors of acute NW after EVT.

\section{Methods}

Data from all consecutive patients treated with EVT for anterior and posterior circulation strokes at our comprehensive stroke center from 2015 to 2017 were retrospectively collected. The patients were treated with IV tPA if they presented within 3-4.5 h from symptom onset and met the standard criteria for tPA. If there were no contraindications, consent was obtained from a family member or legal representative present, and the patient was taken directly for EVT. NW was defined as increase in NIHSS $\geq 4$ points from the baseline NIHSS in the first $24 \mathrm{~h}$ post EVT.

The selection criteria for EVT were acute ischemic stroke (AIS) with LVO. The treatment window was $6 \mathrm{~h}$ from symptom onset for anterior circulation or beyond $6 \mathrm{~h}$ and/or wake-up strokes based on the patient's clinical and imaging findings as specified in our institute's protocol, and up to $24 \mathrm{~h}$ for posterior circulation strokes. The patients were identified based on brain CT (ASPECTS 6 or more), CT angiogram (presence of LVO), and CT perfusion (with a mismatch ratio of $>1.2$ or core of $<70 \mathrm{~mL}$ ) [8]. In some selected patients, brain 
Table 1. Characteristics of patients with and without neurological worsening

\begin{tabular}{|c|c|c|c|}
\hline Variables & $\begin{array}{l}\text { Without neuro- } \\
\text { worsening }(n=152)\end{array}$ & $\begin{array}{l}\text { With neuro- } \\
\text { worsening }(n=26)\end{array}$ & $p$ value \\
\hline Age, median (IQR) & $63(53-75)$ & $60.5(53-69)$ & 0.50 \\
\hline Male, $n(\%)$ & $93(61.2)$ & $17(65.4)$ & 0.83 \\
\hline Race: whites/AA/others, \% & $38.1 / 33.6 / 28.3$ & $34.6 / 27 / 38.4$ & 0.01 \\
\hline Hypertension, $n(\%)$ & $116(76.3)$ & $22(84.6)$ & 0.45 \\
\hline Diabetes mellitus, $n(\%)$ & $36(23.7)$ & $12(46.2)$ & 0.01 \\
\hline Hyperlipidemia, $n(\%)$ & $55(36.4)$ & $13(50)$ & 0.19 \\
\hline Atrial fibrillation, $n(\%)$ & $33(21.7)$ & $3(11.5)$ & 0.30 \\
\hline Anticoagulation use, $n(\%)$ & $15(9.9)$ & 0 & 0.13 \\
\hline NIHSS, median (range) & $19.5(15-24)$ & $17.5(13-21)$ & 0.19 \\
\hline IV tPA, $n(\%)$ & $148(97.4)$ & $26(100)$ & 1.00 \\
\hline Blood glucose, median (range) & $120.5(106-147)$ & $153(114-203)$ & 0.009 \\
\hline Systolic blood pressure, median (range) & $149(128.5-170)$ & $164(143-189)$ & 0.02 \\
\hline ASPECTS, median (IQR) & $8(7-9)(n=137) *$ & $8(6-9)(n=21)^{*}$ & 0.31 \\
\hline \multicolumn{4}{|l|}{ Collateral score, $n(\%)^{*}$} \\
\hline $0-2 \mathrm{a}$ & $70(63.6)$ & $13(86.7)$ & \multirow[t]{2}{*}{0.09} \\
\hline $2 b-3$ & $40(36.4)$ & $2(13.3)$ & \\
\hline Door to groin puncture time, $\mathrm{h}$ & $1.8(1.4-2.2)$ & $2(1.2-2.5)$ & 0.34 \\
\hline LKW to recanalization, $\mathrm{h}$ & $4.4(3.6-5.5)$ & $4.3(3.3-5.1)$ & 0.41 \\
\hline Recanalization grade, $n(\%)$ & & & 0.34 \\
\hline $0-2 \mathrm{a}$ & $18(11.9)$ & $5(19.2)$ & \\
\hline $2 b-3$ & $133(88.1)$ & $21(80.8)$ & \\
\hline \multicolumn{4}{|l|}{ No. of passes, $n(\%)$} \\
\hline$\leq 3$ & $145(95.3)$ & $24(92.3)$ & \multirow[t]{2}{*}{0.62} \\
\hline$\geq 3$ & $7(4.7)$ & $2(7.7)$ & \\
\hline Angioplasty and stenting, $n(\%)$ & $21(13.8)$ & $3(11.5)$ & 0.05 \\
\hline \multicolumn{4}{|l|}{ Clot location, $n(\%)$} \\
\hline Carotid $\mathrm{T}$ & $26(16.2)$ & $4(15.3)$ & \multirow[t]{5}{*}{0.60} \\
\hline M1 & $71(46.7)$ & $12(46.1)$ & \\
\hline M2 & $19(12.5)$ & $3(11.5)$ & \\
\hline Tandem & $22(14.4)$ & $2(7.6)$ & \\
\hline Basilar & $14(9.2)$ & $5(19.2)$ & \\
\hline \multicolumn{4}{|l|}{ Stroke etiology ${ }^{\S}, n(\%)$} \\
\hline Cardioembolic & $96(63.5)$ & $14(53.8)$ & \multirow[t]{4}{*}{0.30} \\
\hline Large vessel & $22(14.5)$ & 8 (30.7) & \\
\hline Dissection & 18 (11.9) & $2(7.6)$ & \\
\hline Cryptogenic & $15(9.9)$ & $2(7.6)$ & \\
\hline
\end{tabular}

IQR, interquartile range; ASPECTS, Alberta Stroke Program Early CT Score; LKW, last known well. * ASPECTS/collateral score was not measured in all patients due to several reasons noted in the main body of the paper. ${ }^{\S}$ Based on TOAST classification.

MRI was performed if posterior circulation stroke was suspected. The occlusions treated included extracranial internal carotid artery, tandem occlusion, M1 segment of middle cerebral artery (MCA), M2 segment of MCA, and basilar artery.

Baseline demographics of the patients were collected. Admission NIHSS, systolic blood pressure (SBP), and blood glucose (BG) were also recorded. The NIHSS was acquired at the time of presentation and prior to thrombectomy. Intracranial bleeding was classified in hemorrhagic infarction types 1 and 2, and parenchymal hemorrhage types 1 and 2, and subarachnoid hemorrhage. Symptomatic intracranial hemorrhage was defined following ECASS II definition [9].

Time metrics including time from stroke onset, onset to groin puncture, door to groin puncture, and time to recanalization were collected. The use of intra-arterial thrombectomy treatment was recorded, and if patients received any additional interventions besides thrombectomy, such as angioplasty, stenting was 


\begin{tabular}{l|l}
\hline DOI: 10.1159/000499973 & $\begin{array}{l}\text { (c) 2019 S. Karger AG, Basel } \\
\text { www.karger.com/ine }\end{array}$ \\
\hline
\end{tabular}

Soomro et al.: Neuro-Worsening after EVT

Table 2. Multivariable logistic regression analysis of predictors of acute neurological worsening after EVT

\begin{tabular}{lll}
\hline Variables & OR $(95 \% \mathrm{CI})$ & $p$ value \\
\hline Race & $2.10(0.56-7.9)$ & 0.26 \\
Diabetes mellitus & $0.71(0.19-2.6)$ & 0.61 \\
ASPECTS & $1.47(0.12-16.8)$ & 0.75 \\
Collateral score & $3.42(0.69-16.7)$ & 0.12 \\
Admission systolic blood & & \\
$\quad$ pressure & $1.00(0.98-1.02)$ & 0.60 \\
Admission blood glucose & $1.00(1.00-1.01)$ & 0.04 \\
\hline
\end{tabular}

ASPECTS, Alberta Stroke Program Early CT Score.

also recorded. Number of passes were also collected. Reperfusion was assessed by the modified thrombolysis in cerebral infarction (mTICI) score, with TICI $2 \mathrm{~b}$ or 3 considered successful reperfusion. Major procedurerelated complication (including vascular perforation or dissection, distal embolism in a different territory) was collected.

Stroke mechanism according to the TOAST classification were recorded. Clinical outcome was assessed at discharge using modified Rankin scale (mRS); good outcome was defined as discharge mRS 0-2. Final infarct volume (FIV) on DWI sequence at $24 \mathrm{~h}$ were also measured for patient using OsiriX software.

\section{Statistical Analysis}

Descriptive statistics were provided for the baseline clinical, radiographic, and procedural variables (Table 1). Two-sample $t$ test or Wilcoxon rank sum test were used to compare the NW and non-NW group for continuous variables, and Chi-square test and Fisher's exact test were used to compare the two groups for categorical variables. Univariate logistic regression analysis was used to model the probability of NW on individual patient predictors including demographic, clinical, and radiographic variables. Variables with $p$ values less than 0.1 were included in the final multivariable logistic regression model. Finally, the association between the reasons of NW and mRS at discharge were evaluated by Fisher's exact $t$ test. Regression plots were created between change in NIHSS and FIV and mRS at discharge. We also performed receiver operating characteristic analysis on variable(s) that were found to have strong association based on multivariable logistic analysis. All statistical analyses were performed in SAS 9.4 (Cary, NC, USA).

\section{Results}

Of the 178 patients screened, 26 (14.7\%) were identified to have experienced NW in the first $24 \mathrm{~h}$ after EVT. Median NIHSS was 5 (IQR 1-11) for NW in the first $24 \mathrm{~h}$. Median age of our patient population was 63 (IQR 53-74, range 26-89), median baseline NIHSS was 19 (IQR 14-24, range 5-37). There were 18 posterior circulation strokes (10.1\%). Four of the 178 (2.2\%) patients did not receive tPA as they did not meet the standard criteria. Median ASPECTS was 8 (IQR 7-9, range 4-10). In patients with posterior circulation strokes, we did not measure ASPECTS scores or collateral scores (CS).

The baseline characteristics of patients according to NW are presented in Table 1 . The prevalence of atrial fibrillation and use of anticoagulation was higher among patients without NW (21.7 and 9.9\% vs. 11.5 and $0 \%, p=0.30$ and 0.13 ) than with NW. Baseline median SBP was higher among patients with NW than without (164 vs. $149, p=0.02)$. Initial median BG at presentation was also higher in patients with acute NW than without (153 mg/dL vs. 120.5 $\mathrm{mg} / \mathrm{dL}, p<0.01$ ). Median NIHSS (19.5 vs. $17.5, p=0.19$ ), poor CT angiogram pre-thrombectomy CS $0-2$ a (63.7 vs. 86.7\%, $p=0.09$ ), and number of passes $>3$ (4.7 vs. 7.7\%, $p=0.62$ ) statistically did not differ between the two groups. CS was not available in 53 patients due to several reasons such as presence of kidney failure, contrast allergy, or patients who presented as transfers from another facility. Eighteen of those 53 (33.9\%) had posterior circulation 
Fig. 1. Multivariate logistic regression and constructed receiver operating curve from clinical prediction rules.

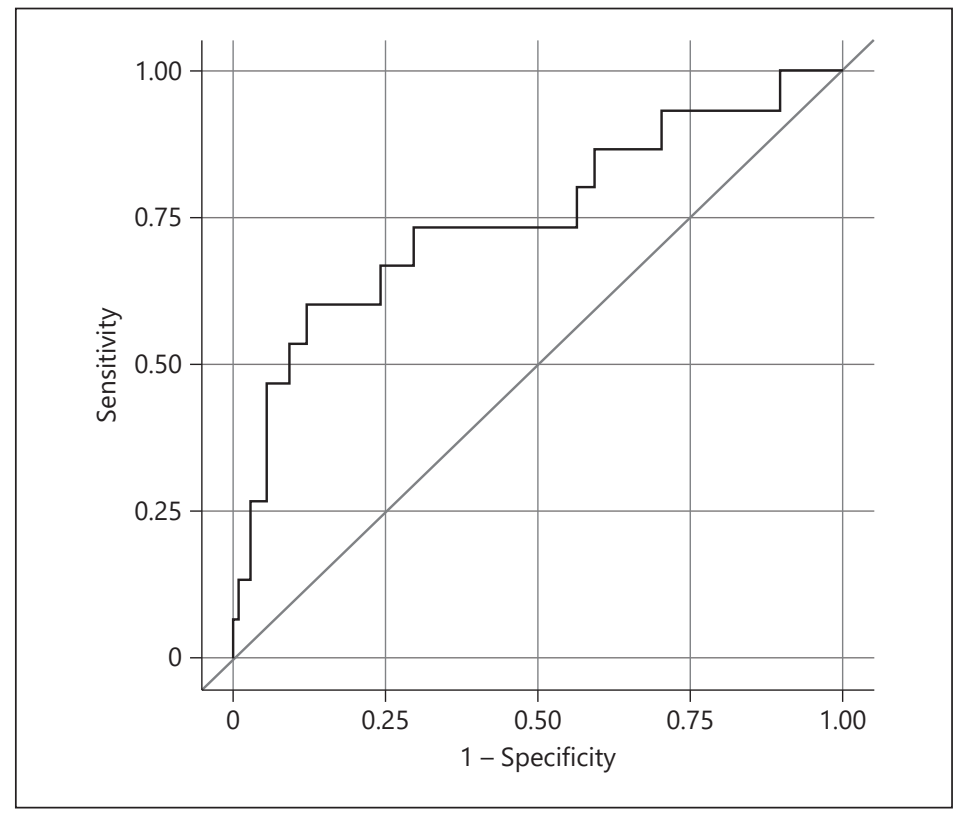

strokes and did not have a CS. Median CT ASPECTS and baseline NIHSS were similar between the two groups. The time from door to groin puncture and time to recanalization were similar between the two groups (Table 1). There were 4 wake-up strokes in the cohort, all belonged to the group without neuro-worsening. Among patients with acute NW, a TICI score <2a was only present in $5(19.2 \%)$ patients. Successful reperfusion (TICI 2 b or 3 ) occurred in $49.7 \%$ of patients in our cohort. Out of 26 patients in the NW group, 21 (80.8\%) achieved successful recanalization (TICI 2b or 3). Univariate analysis showed race, low ASPECTS, poor CS, and prevalence of diabetes mellitus (DM) and presence of higher admission BG and higher initial SBP were associated with NW. In our multivariate analysis, we found admission BG was associated with acute NW (OR 1, CI 1.0-1.01, $p=0.04$ ) (Table 2). Based on multivariate logistic regression and constructed receiver operating curve from clinical prediction rules (graph), which showed an area under the curve of 0.75 , a modest accuracy of predicting acute NW based on the above variables was found (Fig. 1).

Strong correlation was found between change in NIHSS at $24 \mathrm{~h}$ and FIV and mRS at discharge (Fig. 2). Median FIV was higher in the acute NW group (114 $\mathrm{cm}^{3}$, IQR 20.7-150) versus the non-NW group $\left(29 \mathrm{~cm}^{3}\right.$, IQR 8.6-66.8) ( $\left.p=0.01\right)$. Mortality was higher in the NW group, with $46.1 \%$ died during the hospitalization compared to the non-NW group with $10 \%$ $(p<0.001)$ (Fig. 3). Among patients in the NW group, 2 patients had re-occlusion and 0 patient had emboli to new territory.

\section{Discussion}

The current retrospective study is one of the first studies to evaluate acute NW after EVT in the first $24 \mathrm{~h}$. In this single retrospective study, we observed acute NW occurred in $14.7 \%$ of patients. Acute NW after EVT in AIS in our cohort was associated with poor outcome at discharge (MRS 3-6) $(p<0.01)$. African American race, low ASPECTS, poor CS, history of DM, high admission SBP, and high initial BG were associated with NW in our cohort. High admission BG was found to be the only predictor of acute NW. 


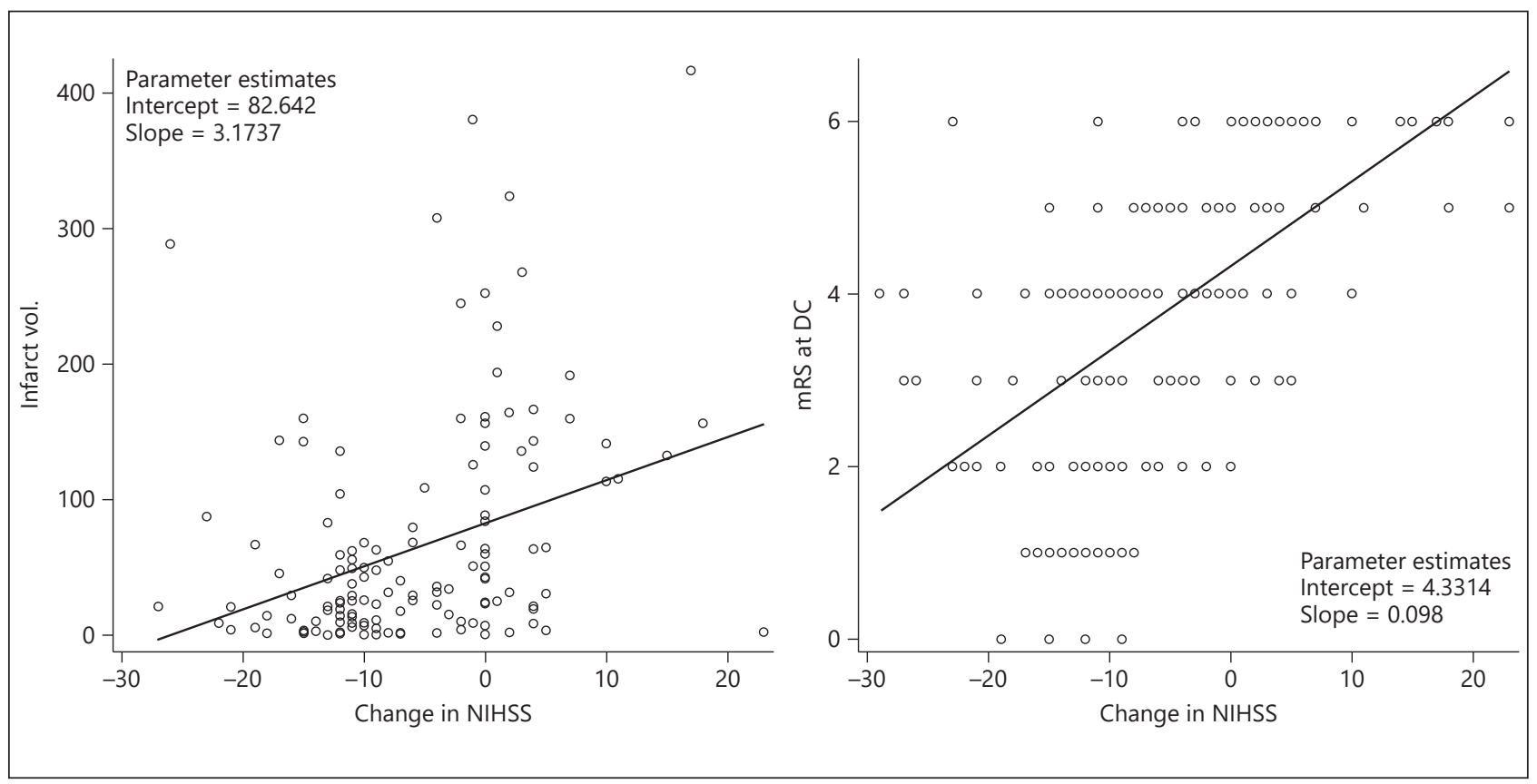

Fig. 2. Regression plots for change in NIHSS with final infarct volume and change in NIHSS with modified Rankin score (mRS) at discharge (DC).

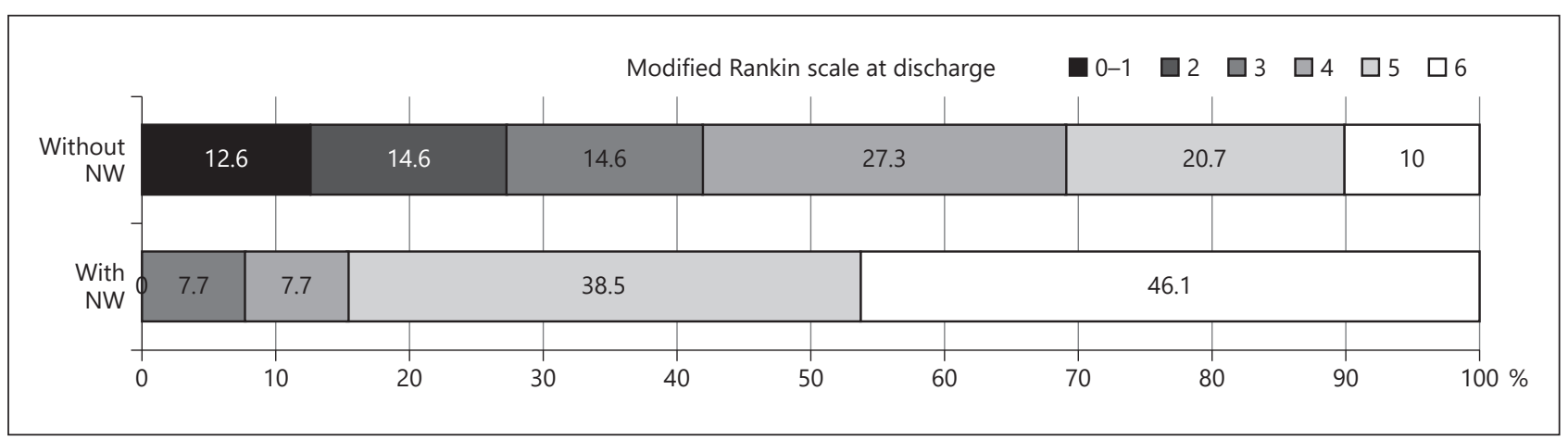

Fig. 3. Modified Rankin score at discharge: distribution between the two groups.

Initial admission hyperglycemia in AIS patients is often associated with worse outcome and even mortality [10]. Unfortunately, almost $40 \%$ of stroke patients present with hyperglycemia [11]. Hyperglycemia after acute stroke may be attributable to several underlying mechanisms, which include a nonspecific reaction to acute stress, autonomic, hormonal, and metabolic alteration as a result of tissue injury, and activation of the hypothalamo-hypophyseal adrenal axis. In animal studies, it has been shown that hyperglycemia can lead to an increase in infarct size. The possible mechanisms of glucose-mediated increase in cerebral infarct size include poor blood flow to the ischemic penumbra, changes in cerebral metabolism, increase in N-methyl-D-aspartate receptor-mediated calcium into the neurons, increase in local edema, and most importantly, glucose-mediated increases in oxidative stress and inflammation [12]. In our study, DM and hyperglycemia were found to be associated with acute NW; however, the DM association was lost in multivariable analysis due to our small sample size. Hyperglycemia without pre-existing DM is also linked to mortality and morbidity in stroke patients 
[13]. BG at admission level of $\geq 116 \mathrm{mg} / \mathrm{dL}$ was an independent predictor of poor outcome in patients without diabetes [14].

In previous studies, lower ASPECTS was associated with risk of intracranial hemorrhage, poor neurological outcomes, and increased mortality after endovascular treatment of AIS [15]. In our study patients with acute NW post thrombectomy, the median ASPECTS was the same in both groups. However, in univariate analysis, ASPECTS showed a trend towards significance and was associated with NW (OR 0.27, CI 0.06-1.19, $p=0.08$ ). High NIHSS on admission previously has been correlated with poor outcomes after thrombolysis and or EVT [16]. In our study, both groups had high median NIHSS on admission; it was relatively higher in the group without NW but not statistically significant.

Collateral flow may influence the risk of hemorrhagic transformation after reperfusion treatment in patients with ischemic stroke. Patients with good collaterals have a lower risk of hemorrhagic transformation. Collateral status can predict the FIV and clinical outcomes after revascularization treatment [17]. Poor collateral status (0-2a) was not statistically different between the two groups.

The time from onset to recanalization $(<5 \mathrm{~h})$ was almost similar between the two groups. Shorter recanalization time has been associated with the absence of infarct growth and improved functional outcomes [18]. Acute internal carotid artery occlusions and vertebrobasilar occlusions are known to be associated with poor outcomes. The correlation may result from a high initial infarct volume with proximal arterial occlusion and technical difficulties in recanalization, resulting in longer time to achieve the occluded vessel. In our study patients in the NW group, 3 patients had a basilar occlusion, 3 had a carotid T occlusion, and 3 patients received angioplasty and stenting ( 1 vertebral, $1 \mathrm{MCA}$, and 1 carotid). About $81 \%$ of the patients in the acute NW group achieved successful recanalization (TICI $2 \mathrm{~b}-3$ ), only $19 \%$ of patients had unsuccessful recanalization. Despite achieving full recanalization, these patients worsened and ultimately had poor outcomes. Number of passes $>3$ has been identified as a predictor of poor outcomes in previous studies. In our cohort of patients with acute worsening, only one patient had $>3$ passes.

Blood pressure is largely a surrogate for cerebral perfusion pressure and has been shown to affect clinical outcomes in AIS patients. Outcomes are generally worse in those who present with either low or severely elevated blood pressure. Elevated blood pressure has been found to be associated with hemorrhagic transformation of cerebral infarction [19]. In our study, patients with NW had significantly higher median blood pressures (initial SBP of $164 \mathrm{~mm} \mathrm{Hg}$ ) compared with the group that did not have worsening (median initial SBP 149).

The most common reason for acute NW in our cohort was found to be progression of the stroke $(42.3 \%)$. The second common reason was reperfusion injury with parenchymal hematoma type $2(26.9 \%)$. Only 2 patients had re-occlusion of the parent vessel that led to worsening in our study.

Our study has multiple limitations, the most important of which are the retrospective study design that is prone to selection bias and the small heterogeneous sample size. Another limitation is that we did not use any independent reviewers to adjudicate recanalization grades in our study. This is a single-center retrospective analysis and caution should be exercised in generalizing the results obtained in our study.

\section{Conclusion}

We found admission BG to be an independent predictor of acute NW after EVT in our study. This finding helps us identify which patients might worsen after EVT and how we can optimize this treatment modality in AIS patients. We are planning a prospective study to test whether better BG control in acute stroke patients reduces NW in those who undergo EVT for LVO. 


\section{Statement of Ethics}

This study was approved by the local institutional review board.

\section{Disclosure Statement}

Drs. Soomro, Zhu, Savitz, and Sarraj have no conflicts of interests and no disclosures.

\section{References}

1 Kircher C, Kreitzer N, Adeoye O. Pre and intrahospital workflow for acute stroke treatment. Curr Opin Neurol. 2016 Feb;29(1):14-9.

2 Mokin M, Sonig A, Sivakanthan S, Ren Z, Elijovich L, Arthur A, Goyal N, Kan P, Duckworth E, Veznedaroglu E, Binning MJ, Liebman KM, Rao V, Turner RD 4th, Turk AS, Baxter BW, Dabus G, Linfante I, Snyder KV, Levy EI, Siddiqui AH. Clinical and Procedural Predictors of Outcomes From the Endovascular Treatment of Posterior Circulation Strokes. Stroke. 2016 Mar;47(3):782-8.

3 Yoon W, Kim SK, Park MS, Baek BH, Lee YY. Predictive Factors for Good Outcome and Mortality After StentRetriever Thrombectomy in Patients With Acute Anterior Circulation Stroke. J Stroke. 2017 Jan;19(1):97-103.

4 Wee CK, McAuliffe W, Phatouros CC, Phillips TJ, Blacker D, Singh TP, et al. Outcomes of Endovascular Thrombectomy with and without Thrombolysis for Acute Large Artery Ischaemic Stroke at a Tertiary Stroke Centre. Cerebrovasc Dis Extra. 2017;7(2):95-102.

5 Tudor G. Jovin, Angel Chamorro, Erik Cobo, María A. de Miquel, Carlos A. Molina, Alex Rovira, Luis San Romá, Joaquín Serena, Sonia Abilleira, Marc Ribó, Mònica Millán, Xabier Urra, Pere Cardona, Elena López-Cancio, Alejandro Tomasello, Carlos Castaño, Jordi Blasco, Lucía Aja, Laura Dorado, Helena Quesada, Marta Rubiera, María Hernandez-Pérez, Mayank Goyal, Andrew M. Demchuk, Rüdiger von Kummer, Miquel Gallofré, Antoni Dávalos. Thrombectomy within 8 hours after Symptom onset in Ischemic Stroke. N Engl J Med. 2015;372: 2296-306.

6 Seners P, Turc G, Oppenheim C, Baron JC. Incidence, causes and predictors of neurological deterioration occurring within $24 \mathrm{~h}$ following acute ischaemic stroke: a systematic review with pathophysiological implications. J Neurol Neurosurg Psychiatry. 2015 Jan;86(1):87-94.

7 Hao Y, Zhang Z, Zhang H, Xu L, Ye Z, Dai Q, et al. Risk of Intracranial Hemorrhage after Endovascular Treatment for Acute Ischemic Stroke: Systematic Review and Meta-Analysis. Intervent Neurol. 2017 Mar;6(1-2):57-64.

8 Campbell BC, Mitchell PJ, Kleinig TJ, Dewey HM, Churilov L, Yassi N, et al.; EXTEND-IA Investigators. Endovascular therapy for ischemic stroke with perfusion-imaging selection. N Engl J Med. 2015 Mar;372(11):1009-18.

9 Hacke W, Kaste M, Fieschi C, von Kummer R, Davalos A, Meier D, et al.; Second European-Australasian Acute Stroke Study Investigators. Randomised double-blind placebo-controlled trial of thrombolytic therapy with intravenous alteplase in acute ischaemic stroke (ECASS II). Lancet. 1998 Oct;352(9136):1245-51.

10 Ozdemir O, Giray S, Arlier Z, Baș DF, Inanc Y, Colak E. Predictors of a Good Outcome after Endovascular Stroke Treatment with Stent Retrievers. ScientificWorldJournal. 2015;2015:403726.

11 Quinn TJ, Dawson J, Walters MR. Sugar and stroke: cerebrovascular disease and blood glucose control. Cardiovasc Ther. 2011 Dec;29(6):e31-42.

12 Garg R, Chaudhuri A, Munschauer F, Dandona P. Hyperglycemia, insulin, and acute ischemic stroke: a mechanistic justification for a trial of insulin infusion therapy. Stroke. 2006 Jan;37(1):267-73.

13 Melamed E. Reactive hyperglycaemia in patients with acute stroke. J Neurol Sci. 1976 Oct;29(2-4):267-75.

14 Natarajan SK, Dandona P, Karmon Y, Yoo AJ, Kalia JS, Hao Q, Hsu DP, Hopkins LN, Fiorella DJ, Bendok BR, Nguyen TN, Rymer MM, Nanda A, Liebeskind DS, Zaidat O0, Nogueira RG, Siddiqui AH, Levy EI. Prediction of adverse outcomes by blood glucose level after endovascular therapy for acute ischemic stroke. J Neurosurg. 2011 Jun;114(6):1785-99.

15 Yoo AJ, Zaidat 00, Chaudhry ZA, Berkhemer OA, González RG, Goyal M, et al.; Penumbra Pivotal and Penumbra Imaging Collaborative Study (PICS) Investigators. Impact of pretreatment noncontrast CT Alberta Stroke Program Early CT Score on clinical outcome after intra-arterial stroke therapy. Stroke. 2014 Mar;45(3):746-51.

16 Yoon W, Kim SK, Park MS, Baek BH, Lee YY. Predictive Factors for Good Outcome and Mortality After StentRetriever Thrombectomy in Patients With Acute Anterior Circulation Stroke. J Stroke. 2017 Jan;19(1):97-103.

17 Marks MP, Lansberg MG, Mlynash M, Olivot JM, Straka M, Kemp S, McTaggart R, Inoue M, Zaharchuk G, Bammer R, Albers GW; Diffusion and Perfusion Imaging Evaluation for Understanding Stroke Evolution 2 Investigators. Effect of collateral blood flow on patients undergoing endovascular therapy for acute ischemic stroke. Stroke. 2014 Apr;45(4):1035-9.

18 Simonsen CZ, Mikkelsen IK, Karabegovic S, Kristensen PK, Yoo AJ, Andersen G. Predictors of Infarct Growth in Patients with Large Vessel Occlusion Treated with Endovascular Therapy. Front Neurol. 2017 Oct 30;8:574.

19 Ozdemir O, Giray S, Arlier Z, Baș DF, Inanc Y, Colak E. Predictors of a Good Outcome after Endovascular Stroke Treatment with Stent Retrievers. ScientificWorldJournal. 2015;2015:403726. 